\title{
Busbar arcs at large Fusion Magnets: Model Experiments on Busbar arcing in a Double Walled Feeder Tube with the LONGARC device
}

Dmitry Klimenko, Volker Pasler

Objective: Model arc experiments were set up to study the propagation and and DEMO
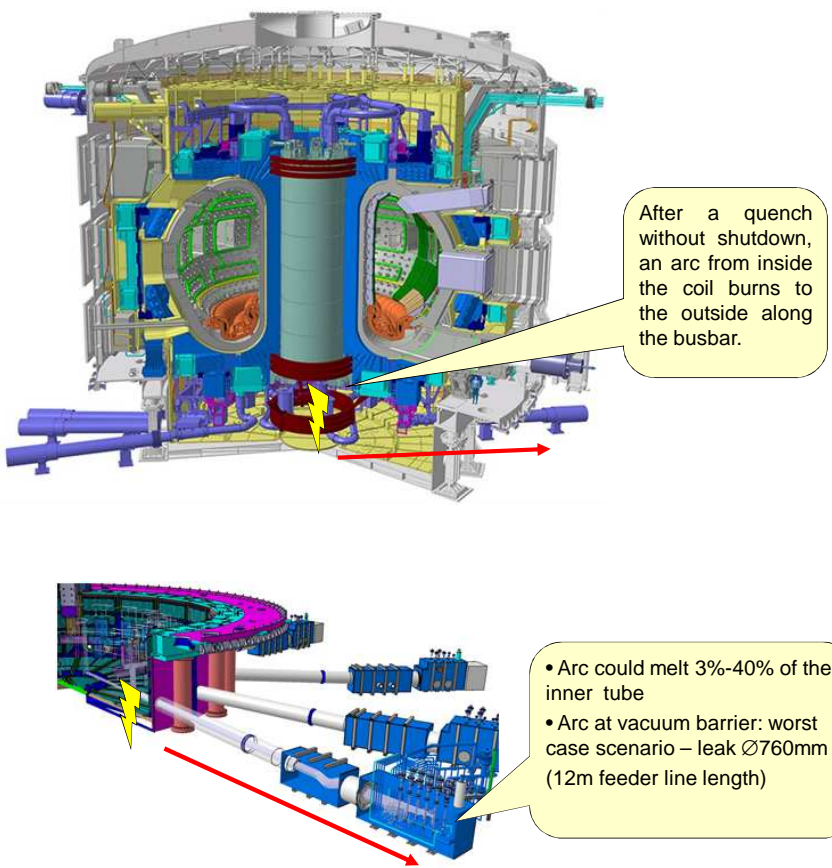

- Arc could melt $3 \%-40 \%$ of the inner tube

- Arc at vacuum barrier: worst case scenario - leak $\varnothing 760 \mathrm{~mm}$ (12m feeder line length)

\section{Purposes of the Experiments:}

Accident scenario with inductively driven high current arcs at Toroidal Field (TF) magnet coil busbars that propagate to the cryostat wall and penetrate it

Support of numerical model development

- Model validation damage potential of possible high current arcs at busbars of tokamak devices such as ITER

\section{LONGARC setup for experiments with double walled feeder tube: Inline mode}

The present work is to simulate the structure side arc spot in geometry similar an busbar feeder tube.
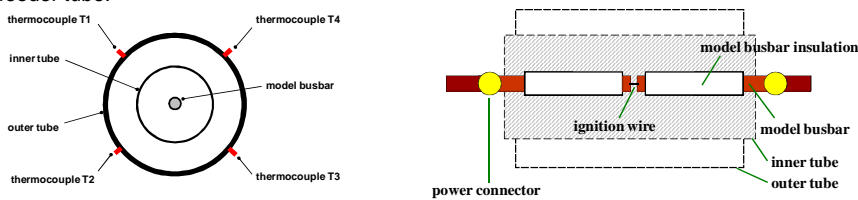

Cut view of setup with model busbar with double tube feeder equipped with 4 thermocouples

Setup for inline arcing with double tube

\section{Results: tube damage}

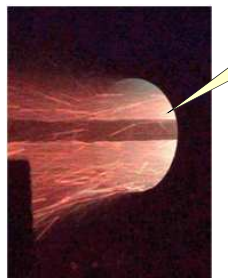

Arc burns in inner
tube only

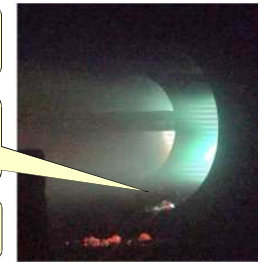

Double walled feeder, equipped with thermocouples after an arc experiment. Outer tube is in good condition
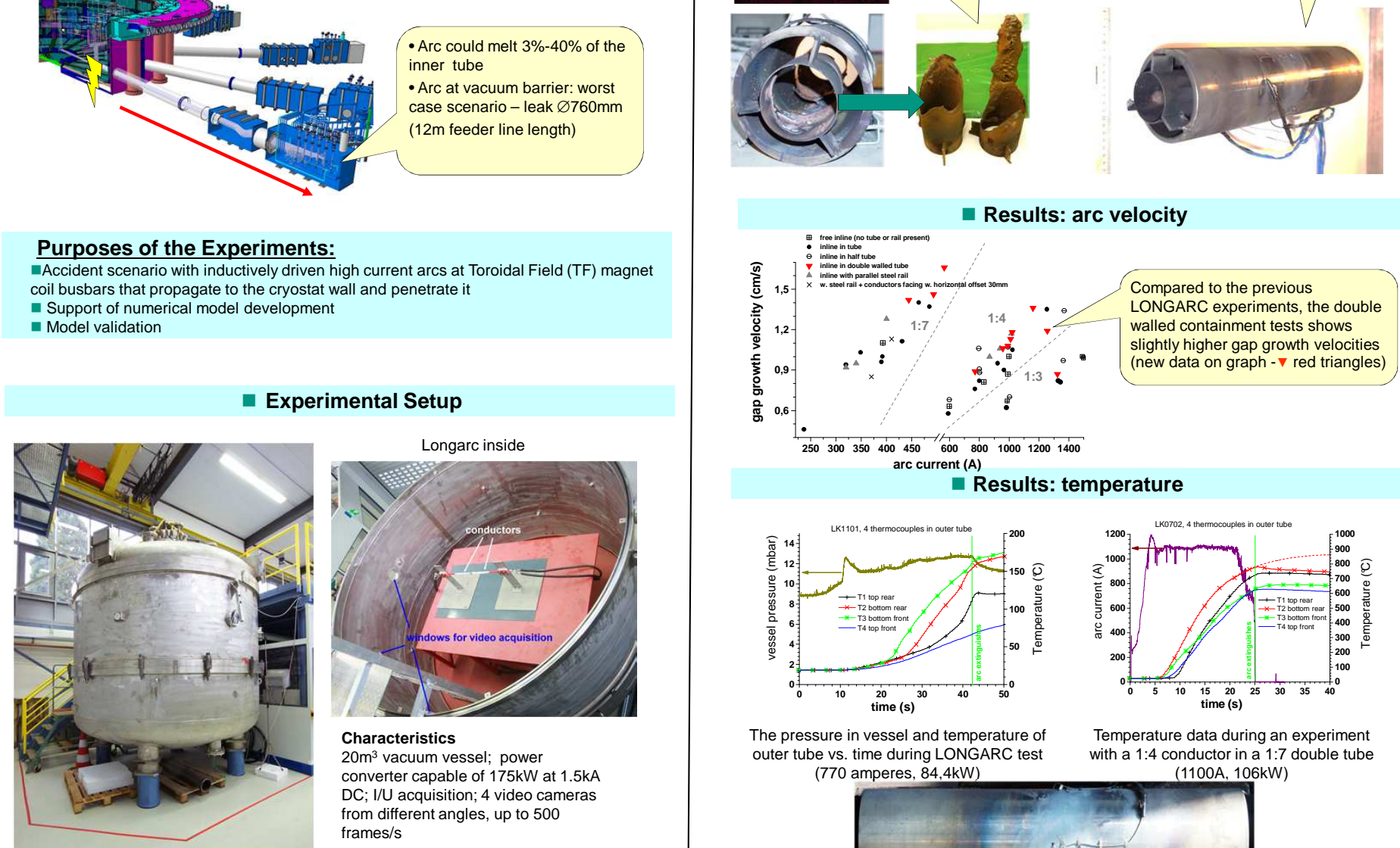

Characteristics

$20 \mathrm{~m}^{3}$ vacuum vessel; power converter capable of $175 \mathrm{~kW}$ at $1.5 \mathrm{kA}$ DC; I/U acquisition; 4 video cameras from different angles, up to 500 frames/s

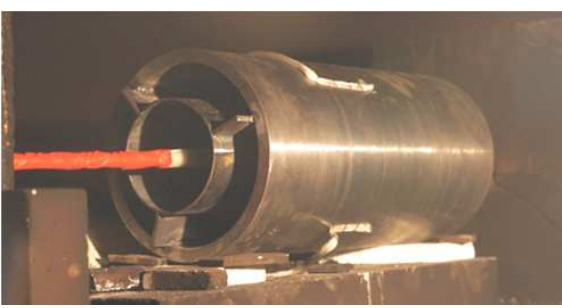

Setup of a double walled feeder line carrying a single model busbar.

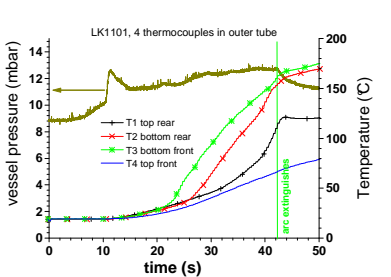

The pressure in vessel and temperature of outer tube vs. time during LONGARC test (770 amperes, 84.4kW)

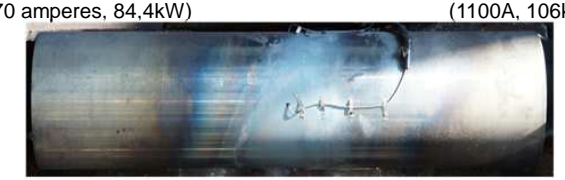

Tempering colours on the outer tube after an experiment (1100A, 106kW) with a 1:4 conductor

\section{- Conclusions}

- The LONGARC device was successfully implemented for busbar to double walled feeder tubes arcing model experiments. the inner tube than comparable single inner tube experiments.

- The outer tube of busbars is expected to withstand high current arcing in any case.

- For DEMO, the double walled TF feeder tube concept of ITER seems a very promising approach to avoid holes in the cryostat vacuum caused by a moving busbar arc along the feeder region. in a $1: 7$ double tube

The double tube experiments in LONGARC device did show slightly more severe damage to 